\title{
Nonlinear controls for a class of discrete-time bilinear systems
}

\author{
Min-Shin Chen ${ }^{1, *, \dagger}$, Yean-Ren Hwang ${ }^{2,}$ and Kun-Chi Huang ${ }^{1}$ \\ ${ }^{1}$ Department of Mechanical Engineering, National Taiwan University, Taipei 106, Taiwan, R. O.C \\ ${ }^{2}$ Department of Mechanical Engineering, National Central University, Chung-Li 310, Taiwan, R.O.C
}

\begin{abstract}
SUMMARY
For a discrete-time neutrally stable bilinear system, a nonlinear state feedback control based on the passivity design has been proposed to stabilize the system globally and asymptotically. This paper shows that the decay rate resulting from the passivity control is not exponential, and the system's response speed becomes very sluggish asymptotically. A 'normalized' nonlinear control is therefore proposed to achieve exponential stability. The new exponentially stabilizing control not only improves the system's response speed, but also enhances the system's robustness against small parametric perturbations. Copyright (C) 2003 John Wiley \& Sons, Ltd.

KEY WORDS: bilinear system; discrete-time system; exponential stability; asymptotical stability; robustness
\end{abstract}

\section{INTRODUCTION}

Bilinear systems comprise an important subclass of nonlinear dynamical systems because numerous real-world dynamical plants have bilinear structures References [1,2]. Real-world examples include engineering applications in nuclear, thermal, chemical processes, and nonengineering applications in biology, social-economics, immunology and so on. Therefore, it is important to devise efficient control laws for such bilinear systems.

An early treatment of discrete time bilinear system control is a state feedback control design Reference [3] based on hyperstability, which ensures local asymptotical stability. In References $[4,5]$, an output feedback control is proposed for general time-varying bilinear systems, which again ensures only local asymptotic stability. Later, based on the concept of passivity, a state feedback control Reference [6] is proposed for an open-loop stable bilinear system. The design achieves global asymptotic stabilization under a zero-state detectability assumption.

\footnotetext{
*Correspondence to: M Chen National Taiwan University, Taipei 106, Taiwan, R.O.C

†E-mail: mschen@ccms.ntu.edu.tw

${ }^{\S}$ E-mail: yhwang@cc.ncu.edu.tw

Contract/grant sponsor: National Science Council of the Republic of China;

Contract/grant number: NSC89-2213-E-002-087
}

Published online 3 April 2003

Copyright (C) 2003 John Wiley \& Sons, Ltd.
Received 7 September 2001

Revised 29 March 2002

Accepted 7 October 2002 
The objectives of this paper are as follows. First, using the Lyapunov function analysis, one can show that when the bilinear system is homogeneous (corresponding to the case $D=0$ in Reference [6]), the closed-loop decay rate for the passivity control in Reference [6] is not exponential. The norm of the state $\|x(k)\|$ decays as $1 / \sqrt{k}$; as a result, the decay rate becomes sluggish as time goes on. Second, a 'normalized' nonlinear control is therefore proposed in this paper to achieve exponential stability of the closed-loop system. Such a new control not only substantially improves the system response speed, but also enhances robustness of the controlled system. In this paper, the analysis and control design are introduced in detail for single-input bilinear systems, and then roughly sketched for multi-input bilinear systems in the final section.

\section{REFORMULATION OF ASYMPTOTICALLY STABILIZING CONTROL}

Considers a discrete-time homogeneous bilinear system

$$
x(k+1)=A x(k)+u(k) N x(k) \quad x(0)=x_{0}
$$

where $x(t) \in \mathbb{R}^{n}$ is the system state vector, $u(k)$ is a scalar control input, and $A \in R^{n \times n}$ and $N \in R^{n \times n}$ are constant square matrices. The bilinear system (1) is assumed to satisfy the following two assumptions:

\section{Assumption Al}

There exists a positive definite matrix $P>0$ such that

$$
A^{\mathrm{T}} P A-P=0
$$

Assumption A1 restricts that the open-loop system be neutrally stable, and all the eigenvalues of $A$ fall on the unit circle.

Assumption A2

There exists an integer $m>0$ such that

$$
\operatorname{rank}\left[A^{-1} N x, A^{-2} N A x, \ldots, A^{-m} N A^{m-1} x\right]=n
$$

for any non-zero $x$ in $R^{n}$. Assumption A2 [7] guarantees that system (1) is controllable [8] at any non-zero $x$. Notice that under the Assumption A1, the zero-state detectability in Reference [6] is equivalent to the controllability Assumption A2 here.

One now presents the asymptotically stabilizing control introduced in Reference [6]; however, in this paper the control will be constructed based on the Lyapunov function theory instead of on the passivity theory as in Reference [6]. The advantage of using the Lyapunov analysis is to make decay rate analysis possible, as will be shown in Section 3. The asymptotically stabilizing control is as follows:

$$
u(k)=-\rho \frac{a_{x}}{2 b_{x}+\varepsilon}, \quad 0<\rho \leqslant 2, \varepsilon>0
$$

where $\rho$ is the control gain, normally chosen to be one, $\varepsilon$ is a small positive number, and $a_{x}$ and $b_{x}$ are abbreviations of $a_{x(k)}$ and $b_{x(k)}$ :

$$
\begin{aligned}
& a_{x(k)}=2 x^{\mathrm{T}}(k) A^{\mathrm{T}} P N x(k) \\
& b_{x(k)}=[N x(k)]^{\mathrm{T}} P[N x(k)]
\end{aligned}
$$


in which $P$ is the positive definite matrix in (2). Note that $b_{x}$ may be either positive or zero. The introduction of the small parameter $\varepsilon(>0)$ in the control law (4) is to avoid possible division by zero in $u(k)$.

In this section, it will be shown that the bilinear system (1) with the control law (4) is asymptotically stable.

\section{Lemma 1}

Consider the bilinear system (1) with the control law (4). If the bilinear system satisfies Assumptions A1 and A2, and

$$
a_{x(k+i)}=2 x(k+i)^{\mathrm{T}} A^{\mathrm{T}} P N x(k+i)=0, \quad \forall \quad i=0,1,2, \ldots, m-1
$$

then $x(k)$ must be the null vector.

\section{Proof}

Hypothesis (5) can be put into the following matrix form, using the system dynamics $x(k+i)=A^{i} x(k)$ under hypothesis (5),

$$
x^{\mathrm{T}}(k)\left[A^{\mathrm{T}} P N x(k),\left(A^{\mathrm{T}}\right)^{2} P N A x(k), \ldots,\left(A^{\mathrm{T}}\right)^{m} P N A^{m-1} x(k)\right]=0
$$

Since $A^{\mathrm{T}} P=P A^{-1}$ from Assumption A1, (6) becomes

$$
x^{\mathrm{T}}(k) P\left[A^{-1} N x(k), A^{-2} N A x(k), \ldots, A^{-m} N A^{m-1} x(k)\right]=0
$$

If $x(k)$ is non-zero, according to the controllability assumption A2, the matrix in (7) is full rank; hence, $x^{\mathrm{T}}(k) P=0$. Since $P$ is positive, one concludes that $x(k)=0$. One then reaches a contradiction with the assumption that $x(k)$ is non-zero, therefore, $x(k)$ must be zero.

\section{Theorem 1}

The bilinear system (1) with the control law (4) is globally asymptotically stable.

Proof

Choose a Lyapunov function candidate

$$
V(k)=x^{\mathrm{T}}(k) P x(k) \geqslant 0
$$

where $P$ is the positive definite matrix in (2). One can derive, along the trajectory of (1) and (4),

$$
\begin{aligned}
\Delta V(k) & =V(k+1)-V(k) \\
& =2 x^{\mathrm{T}}(k) A^{\mathrm{T}} P N x(k) u(k)+[N x(k)]^{\mathrm{T}} P[N x(k)] u^{2}(k) \\
& =-a_{x}^{2} \frac{\rho\left[(2-\rho) b_{x}+\varepsilon\right]}{\left(2 b_{x}+\varepsilon\right)^{2}} \leqslant 0
\end{aligned}
$$

Since $b_{x} \geqslant 0$ and $2 \geqslant \rho>0, \Delta V(k)$ is always non-positive. From the Lyapunov stability theory [8], the system state $x(k)$ will remain uniformly bounded; that is,

$$
x(k) \in D
$$

for some bounded region $D$ in the state space.

To show that the state $x(k)$ actually approaches zero asymptotically, notice from (8) that $\Delta V(k)=0$ implies $a_{x(k)}=0$. Since the largest invariant set contained in $\left\{x \mid a_{x(k)} \equiv 0\right\}$ is $\{0\}$ 
according to Lemma 1, one concludes from the LaSalle theorem [8] that $x(k)$ approaches zero asymptotically, and hence the controlled system is asymptotically stable.

\section{Remark}

The passivity control $(D=0)$ in Reference [6, Theorem 4] corresponds to control (4) in this paper with $\rho=2$ and $\varepsilon=4$. However, these parameter values are not optimal as far as the decay rate of $V(k)$ is concerned. The reason is as follows. If one sets $\partial \Delta V(k) / \partial u(k)=0$ in the proof of Theorem 1, one obtains

$$
u^{*}(k)=-\frac{a_{x}}{2 b_{x}}, \quad b_{x} \geqslant 0
$$

which will maximize the decrease rate of $V(k)$. Comparing the above $u^{*}(k)$ with control (4) immediately suggests that for fast responses, one should choose $\rho=1$ and $\varepsilon$ sufficiently small in the control law (4). Simulation results do confirm such a choice. Note that $\varepsilon$ is added in the control (4) to avoid the possible division by zero in $u^{*}(k)$.

\section{DECAY RATE ANALYSIS OF ASYMPTOTICALLY STABILIZING CONTROL}

The purpose of this section is to provide a decay rate analysis for the asymptotically stabilizing control (4), which is not available when the control was first proposed in Reference [6]. Define the notations:

$$
a_{e} \triangleq \frac{a_{x}}{\|x\|^{2}}=2 e(k)^{\mathrm{T}} A^{\mathrm{T}} P N e(k) \quad \text { where } \quad e(k) \triangleq \frac{x(k)}{\|x(k)\|}
$$

and $e(k)$ is called the normalized state.

\section{Definition}

Define a scalar real function $B_{1}(e(k),\|x(k)\|): S \times I \rightarrow R^{+} \cup\{0\}$ for the controlled system (1) and (4)

$$
B_{1}(e(k),\|x(k)\|)= \begin{cases}\sum_{i=k}^{k+m-1}\left[a_{e(i)}\right]^{2}=\sum_{i=k}^{k+m-1}\left[2 \frac{x^{\mathrm{T}}(i)}{\|x(i)\|} A^{\mathrm{T}} P N \frac{x(i)}{\|x(k)\|}\right]^{2}, & x(k) \neq 0 \\ \lim _{\|x(k)\| \rightarrow 0} B_{1}(e(k),\|(k)\|), & x(k)=0\end{cases}
$$

where $S$ is the unit sphere in $R^{n}$ and $I=[0, r]$, in which $r=^{\triangle} \max _{i}\{\|x(i)\| \mid x(i) \in D$ in $(9)$, $i=0,1, \ldots, \infty\}$.

From the knowledge of $x(k)$, one can determine consecutively $x(k+1), x(k+2), \ldots, x \times$ $(k+m-1)$, from the closed-loop dynamics (1) and (4):

$$
x(k+1)=\left[A-\rho \frac{2 x^{\mathrm{T}}(k) A^{\mathrm{T}} P N x(k)}{[N x(k)]^{\mathrm{T}} P[N x(k)]+\varepsilon} N\right] x(k)
$$

The value of the function $B_{1}(\cdot, \cdot)$ in (11) can then be exactly calculated; hence, the function $B_{1}(\cdot, \cdot)$ is uniquely defined by $e(k)$ and $\|x(k)\|$. Note that $B_{1}(e(k),\|x(k)\|)$ at the origin $x(k)=0$ is defined by a limiting process, for otherwise division by zero will take place at $x(k)=0$. The next lemma shows that the limit in (11) does exist. 
Lemma 2

For the closed-loop system (1) and (4), there exists a real scalar function $B_{0}(e(k)): S \rightarrow R^{+} \cup\{0\}$ such that

$$
B_{0}(e(k))=\lim _{\|x(k)\| \rightarrow 0} B_{1}(e(k),\|x(k)\|)
$$

Proof

When $x(k)$ approaches the origin, the closed-loop dynamics (12) can be described by its linearized model:

$$
x(k+1)=A x(k) \quad \text { as } \quad\|x(k)\| \rightarrow 0
$$

As a result, there exists a recursive relationship for the normalized state $e(k)$ :

$$
e(k+1)=\frac{A e(k)}{\|A e(k)\|} \quad \text { as } \quad\|x(k)\| \rightarrow 0 .
$$

One can determine the value of $B_{1}(e(k),\|x(k)\|)$, a functions of $e(i), i=k, k+1, \ldots, k+m-1$, using only the information of the initial normalized state $e(k)$ and the recursive relationship (13). In other words, when $x(k)$ is close to the origin, given any $e(k)$, the value of $B_{1}(e(k),\|x(k)\|)$ is independent of $\|x(k)\|$, and hence the limiting function in (11) is well defined.

The following lemma shows that the controllability assumption (3) guarantees that the value of $B_{1}(e(k),\|x(k)\|)$ is bounded above from zero on its domain $S \times I$.

\section{Lemma 3}

For the closed-loop system (1) and (4), there exists some positive constant $\beta$ such that

$$
\inf _{\substack{e(k) \in S \\\|x(k)\| \in I}}\left\{B_{1}(e(k),\|x(k)\|)\right\}=\beta>0
$$

where inf denotes the infimum taken over all $e(k) \in S$ and all $\|x(k)\| \in I$.

Proof

One first shows that $B_{1}(e(k),\|x(k)\|)$ is positive for all $e(k) \in S$ and $\|x(k)\| \in I \backslash\{0\}$ (at every point in its domain except at $x(k)=0)$, then shows that $B_{1}(e(k),\|x(k)\|)$ is actually also positive at $x(k)=0$.

First, consider the case $\|x(k)\| \neq 0$. By definition, $B_{1}(\cdot, \cdot)$ must be non-negative. Hence, to prove that $B_{1}(\cdot, \cdot)$ is positive, one only needs to show that $B_{1}(\cdot, \cdot)$ is non-zero. Assume the contrary: $B_{1}(e(k),\|x(k)\|)$ is zero at some $e(k)$ and some non-zero $x(k)$, Then,

$$
x^{\mathrm{T}}(i) A^{\mathrm{T}} P N x(i) \equiv 0, \quad \forall \quad i=k, k+1, \ldots, k+m-1
$$

Applying Lemma 1 to the above equation suggests that $x(k)=0$, contradicting the hypothesis of this case that $x(k) \neq 0$. Therefore, $B_{1}(\cdot, \cdot)$ must be positive at every point where $x(k) \neq 0$.

Next, consider the case $\|x(k)\|=0$. To prove that $B_{1}(e(k),\|x(k)\|)$ is positive at $\|x(k)\|=0$, recall from the proof of Lemma 2 that, given any $e(k)$, the value of $B_{1}(e(k),\|x(k)\|)$ becomes a constant independent of $\|x(k)\|$ as it approaches zero. Since it is already proved in the first case of this lemma that $B_{1}(e(k),\|x(k)\|)$ is positive no matter how small $\|x(k)\|$ is, this constant must be positive. Hence, from the limiting definition of $B_{1}(e(k),\|x(k)\|)$ at $\|x(k)\|=0$, the value of $B_{1} \times$ $(e(k),\|x(k)\|)$ must be positive at $\|x(k)\|=0$. 
Finally, since $B_{1}(\cdot, \cdot)$ is positive at every point in its domain from the above analysis, and the domain of $B_{1}(\cdot, \cdot), S \times I$, is compact, it follows from Theorem 4.4.1 in Reference [9] that the infimum of $B_{1}(\cdot, \cdot)(=\beta)$ is also positive.

One can now present a decay rate analysis for the Lyapunov function $V(k)$.

Theorem 2

Consider the Lyapunov function $V(k)=x^{\mathrm{T}}(k) P x(k)$ for the closed-loop system (1) and (4). For the integer $m$ as in (3) and for some real positive constant $\pi$, the decay rate of $V(k)$ is given by

$$
V(m k) \leqslant \frac{1}{\pi k} \quad \text { as } \quad k \rightarrow \infty
$$

Proof

Rewrite (8) as

$$
\begin{aligned}
V(k+1)-V(k) & =-\left(\frac{a_{x}}{\|x\|^{2}}\right)^{2} \frac{\rho\left[(2-\rho) b_{x}+\varepsilon\right]}{\left(2 b_{x}+\varepsilon\right)^{2}} \frac{\|x\|^{4}}{\left(x^{T} P x\right)^{2}} V^{2}(k) \\
& \leqslant-a_{e}^{2} \frac{\rho\left[(2-\rho) b_{x}+\varepsilon\right] 1}{\left(2 b_{x}+\varepsilon\right)^{2} \lambda_{P}^{2}} V^{2}(x)
\end{aligned}
$$

where $\lambda_{P}$ is the maximum eigenvalue of $P$. Since $x(k)$ is uniformly bounded from Theorem 1 , one can show that there exists a positive constant $c_{1}$ such that for all $x(k) \in D$ in (9),

$$
\frac{\rho\left[(2-\rho) b_{x}+\varepsilon\right]}{\left(2 b_{x}+\varepsilon\right)^{2}} \geqslant c_{1}>0
$$

Equation (14) then becomes

$$
V(k+1)-V(k) \leqslant-\frac{c_{1}}{\lambda_{P}^{2}} a_{e(k)}^{2} V^{2}(k)
$$

Derive, using (15),

$$
\begin{aligned}
\frac{1}{V(k+1)}-\frac{1}{V(k)} & =\frac{V(k)-V(k+1)}{V(k) V(k+1)} \\
& \geqslant \frac{c_{1}}{\lambda_{P}^{2}} a_{e(k)}^{2} \frac{V(k)}{V(k+1)} \\
& \geqslant \frac{c_{1}}{\lambda_{P}^{2}} a_{e(k)}^{2}
\end{aligned}
$$

where the last inequality results from $V(k+1) \leqslant V(k)$. Summing the above equations from $k$ to $k+m-1$ yields

$$
\frac{1}{V(k+m)}-\frac{1}{V(k)} \geqslant \frac{c_{1}}{\lambda_{P}^{2}} \sum_{i=k}^{k+m-1} a_{e(i)}^{2} \geqslant \frac{c_{1}}{\lambda_{P}^{2}} \beta,
$$


where the second inequality results from Lemma 3. By setting $k=0, m, 2 m, \ldots,(\ell-1) m$ in (16), and summing all the equations, one has

$$
\frac{1}{V(\ell m)}-\frac{1}{V(0)} \geqslant c_{1} \frac{\beta}{\lambda_{P}^{2}} \ell
$$

therefore, $V(\ell m) \leqslant V(0) /\left(c_{1}\left(\beta / \lambda_{P}^{2}\right) \ell V(0)+1\right)$, which, when $\ell$ is replaced by $k$ and $c_{1}\left(\beta / \lambda_{P}^{2}\right)$ by $\pi$, proves the claim of the theorem.

\section{Remark}

Theorem 2 shows that the passivity control (4) in Reference [6] results in a power-law decay rate. With the power-law decay rate, the system response slows down substantially as $k$ approaches infinity, making control (4) unacceptable in real-world applications. This motivates a new control design that can produce a faster decay in the next section.

\section{EXPONENTIALLY STABILIZING CONTROL}

As a modification of the asymptotically stabilizing control (4), the following exponentially stabilizing control is proposed for system (1):

$$
u(k)=-\rho \frac{a_{e}}{2 b_{e}+\varepsilon}, \quad 0<\rho \leqslant 2, \varepsilon>0
$$

where $\rho$ is the control gain, normally one, $\varepsilon$ is a small positive number to avoid division by zero, and

$$
\begin{aligned}
& a_{e(k)}=2 e^{\mathrm{T}}(k) A^{\mathrm{T}} P N e(k) \\
& b_{e(k)}=[N e(k)]^{\mathrm{T}} P[N e(k)]
\end{aligned}
$$

in which $e(k)$ is the normalized state defined in (10). The above exponentially stabilizing control law (17) has exactly the same structure as the asymptotically stabilizing control law (4) studied in the previous section except that the system state $x(k)$ in (4) is now replaced by the "normalized" state $e(k)$ in the new control.

The following lemmas are required for the stability analysis of the proposed new control.

\section{Lemma 4}

Consider the bilinear system (1) and (17). If

$$
a_{e(k+i)}=2 e(k+i)^{\mathrm{T}} A^{\mathrm{T}} P N e(k+i)=0, \quad \forall i=0,1,2, \ldots, m-1
$$

where $e(k)$ is defined in $(10)$, then $x(k)$ must be the null vector.

Proof

Assume that $x(k)$ is non-zero. Multiplying (18) by $\|x(k)\|^{2}$ results in (5). The rest of proof then follows that of Lemma 1.

Next, define a scalar function:

$$
B_{2}(e(k))=\sum_{i=k}^{k+m-1}\left[a_{e(i)}\right]^{2}=\sum_{i=k}^{k+m-1}\left[2 e^{\mathrm{T}}(i) A^{\mathrm{T}} P N e(i)\right]^{2}
$$


for the new closed-loop system (1) and (17):

$$
x(k+1)=\left[A-\rho \frac{2 e^{\mathrm{T}}(k) A^{\mathrm{T}} P N e(k)}{[N e(k)]^{\mathrm{T}} P[N e(k)]+\varepsilon} N\right] x(k)=^{\Delta} f(x(k))
$$

Note that the right-hand side of the function $B_{2}(\cdot)$ in (19) is almost the same as that of $B_{1}(\cdot, \cdot)$ in (11), but the two functions are defined differently. First, $B_{2}(\cdot)$ is not defined at $x(k)=0$, while $B_{1}(\cdot, \cdot)$ is. Second, $B_{2}(\cdot)$ is a function of $e(k)$ only, while $B_{1}(\cdot, \cdot)$ a function of both $\|x(k)\|$ and $e(k)$. The reason why $B_{2}(\cdot)$ in (19) is defined a function of $e(k)$ only is as follows. Notice that $f(x(k))$ in (20) satisfies the so-called homogeneous property for all $\|x(k)\|$ (not just for $\|x(k)\| \rightarrow 0$ as shown in Lemma 2 for $\left.B_{1}(\cdot, \cdot)\right)$; that is,

$$
f(r x(k))=r f(x(k)) \quad \text { for all } r>0
$$

Based on (20) and (21), it can be deduced that there exists a continuous function $F(\cdot): S \rightarrow S$, where $S$ is the unit sphere in $R^{n}$, such that

$$
e(k+1)=F(e(k)), \quad F(e(k))={ }^{\Delta} \frac{f(e(k))}{\|f(e(k))\|} .
$$

Using relation (22), one can calculate $B_{2}(\cdot)$ based on the knowledge of $e(k)$ only. Hence, it is not a function of state norm.

By following the same procedure as in the proof of Lemma 3, and using Lemma 4, one can show that the non-negative function $B_{2}(\cdot)$ is actually bounded above from zero for all $e(k) \in S$; i.e., for all non-zero $x(k) \in R^{n}$.

\section{Lemma 5}

For the closed-loop system (1) and (17), there exists a constant $\alpha>0$ such that

$$
\inf _{e(k) \in S}\left[B_{2}(e(k))\right]=\alpha>0
$$

One can now establish the exponential stability for the proposed new control law.

\section{Theorem 3}

If the bilinear system (1) satisfies the Assumptions A1 and A2, the nonlinear control law (17) stabilizes the system exponentially.

Proof

Define a Lyapunov function candidate $V(k)=x^{\mathrm{T}} P x(k)$, where $P$ is as in (2). The increment of $V(k)$ along (1) and (17) is given by

$$
\begin{aligned}
& V(k+1)-V(k) \\
= & x^{\mathrm{T}}(k+1) P x(k+1)-x^{\mathrm{T}}(k) P x(k) \\
= & 2 x^{\mathrm{T}}(k) A^{\mathrm{T}} P N x(k) u(k)+(N x(k))^{\mathrm{T}} P(N x(k)) u(k)^{2} \\
= & -a_{e(k)}^{2} \frac{\rho\left[(2-\rho) b_{e(k)}+\varepsilon\right]\|x(k)\|^{2}}{\left(2 b_{e(k)}+\varepsilon\right)^{2} x^{T}(k) P x(k)} V(k)
\end{aligned}
$$


Since $b_{e(k)}$ is uniformly bounded, one has

$$
\frac{\rho\left[(2-\rho) b_{e(k)}+\varepsilon\right]}{\left(2 b_{e(k)}+\varepsilon\right)^{2}} \geqslant c>0
$$

for some positive constant $c$. The last inequality in $\Delta V(k)$ can then be deduced to

$$
V(k+1)-V(k) \leqslant-a_{e(k)}^{2} \frac{c}{\lambda_{P}} V(k) \leqslant 0
$$

where $\lambda_{P}$ is the maximum eigenvalue of the positive definite matrix $P$. Since $V(k)$ is nonincreasing, one has

$$
V(k+i) \geqslant V(k+m), \quad i=0,1, \ldots, m-1
$$

List the equations in (23) from $k$ to $k+m-1$, and utilize (24),

$$
\begin{gathered}
V(k+1)-V(k) \leqslant-a_{e(k)}^{2} \frac{c}{\lambda_{P}} V(k) \leqslant-a_{e(k)}^{2} \frac{c}{\lambda_{P}} V(k+m) \\
V(k+2)-V(k+1) \leqslant-a_{e(k+1)}^{2} \frac{c}{\lambda_{P}} V(k+1) \leqslant-a_{e(k+1)}^{2} \frac{c}{\lambda_{P}} V(k+m) \\
(k+m)-V(k+m-1) \leqslant v-a_{e(k+m-1)}^{2} \frac{c}{\lambda_{P}} V(k+m-1) \leqslant-a_{e(k+m-1)}^{2} \frac{c}{\lambda_{P}} V(k+m)
\end{gathered}
$$

Adding the above inequalities together, and quoting Lemma 5, one obtains

$$
V(k+m)-V(k) \leqslant-\left[\begin{array}{ll}
\sum_{i=k}^{k+m-1} & a_{e(i)}^{2}
\end{array}\right] \frac{c}{\lambda_{P}} V(k+m) \leqslant-\frac{\alpha c}{\lambda_{P}} V(k+m)
$$

Rearranging the last inequality gives

$$
V(k+m) \leqslant \frac{1}{1+\alpha c / \lambda_{P}} V(k)
$$

and hence,

$$
V(k m) \leqslant \sigma^{k} V(0) \quad \text { where } \quad \sigma=\frac{1}{1+\alpha c / \lambda_{P}}<1
$$

One concludes from (25) that the Lyapunov function $V(k)$ converges exponentially to zero, and so does $x(k)$ by the definition of $V(k)$.

Remark

Theorem 3 proves that the proposed control (17) produces an exponential deay rate. The exponential decay rate in Theorem 3 is superior to the power-law decay rate in Theorem 2 in two ways. (1) The exponential decay rate is much faster than the power-law decay rate as the time gets large. A clear demonstration of this point is shown in Figure 1 below. (2) When the closedloop system is exponentially stable, it is robust against small unmodelled nonlinearities and small parametric perturbations. For a proof of this, please refer to Reference [10, Theorem 121].

\section{MULTI-INPUT BILINEAR SYSTEMS}

In the previous section, the exponentially stabilizing control is introduced for the single-input case. In this section, the multi-input case will be discussed. In particular, a two-input case will be 


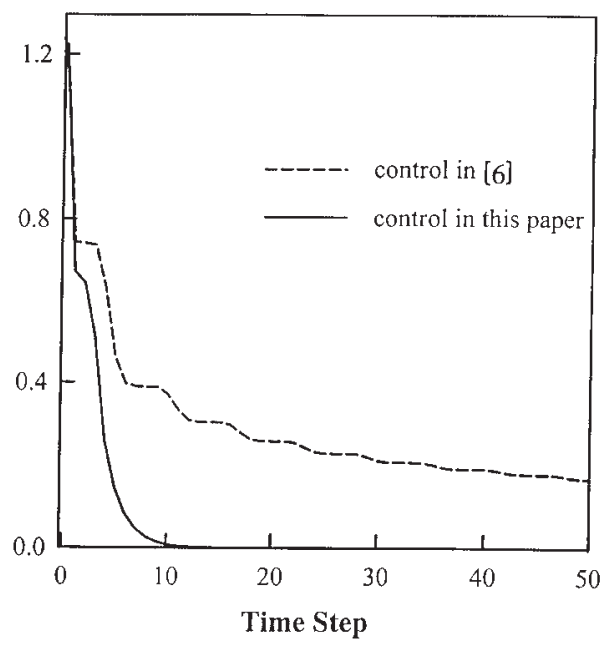

Figure 1. $\|x(t)\|$ for different controls.

examined. Consider the following two-input bilinear system:

$$
x(k+1)=A x(k)+u_{1}(k) N_{1} x(k)+u_{2}(k) N_{2} x(k) \quad x(0)=x_{0}
$$

where $x(k) \in R^{n}$ is the system state vector, $u_{1}(k)$ and $u_{2}(k)$ are two scalar control inputs, and $A$, $N_{1}, N_{2} \in R^{n \times n}$ are constant square matrices. The multi-input bilinear system (26) is assumed to satisfy the same neutral stability assumption A1 in (2) and the multi-input controllability assumption below.

Assumption A2'

There exists an integer $m>0$ such that

$$
\begin{aligned}
& \operatorname{rank}\left[A^{-1} N_{1} x, A^{-2} N_{1} A x, \ldots, A^{-m} N_{1} A^{m-1} x\right. \\
& \left.A^{-1} N_{2} x, A^{-2} N_{2} A x, \ldots, A^{-m} N_{2} A^{m-1} x\right]=n
\end{aligned}
$$

for any non-zero $x$ in $R^{n}$.

An exponentially stabilizing control for the above system is as follows.

$$
U(k)=\left[\begin{array}{l}
u_{1}(k) \\
u_{2}(k)
\end{array}\right]=-\rho\left[N_{e}^{\mathrm{T}}(k) P N_{e}(k)+\varepsilon I\right]^{-1} N_{e}^{\mathrm{T}}(k) P A e(k), \rho \in(0,2]
$$

where $\rho \in(0,2]$ is a positive control gain, $P$ is the positive definite matrix in $(2), N_{e}=\left[N_{1} e \times\right.$ $\left.(k), N_{2} e(k)\right]$, and $e(k)$ the normalized state in $(10)$.

The stability analysis for the multi-input control law follows exactly the single-input case, and is omitted here. Instead, a simulation example is used to verify the effectiveness of the proposed control design. 


\section{Example}

Consider a three-dimensional bilinear system (26) with

$$
A=\left[\begin{array}{rrr}
0.7500 & 0.4330 & 0.5000 \\
0.5000 & -0.8660 & 0 \\
-0.4330 & -0.2500 & 0.8660
\end{array}\right], \quad N_{1}=\left[\begin{array}{lll}
0 & 0 & 0 \\
0 & 0 & 0 \\
0 & 0 & 1
\end{array}\right], \quad N_{2}=\left[\begin{array}{lll}
0 & 0 & 0 \\
0 & 0 & 1 \\
0 & 0 & 0
\end{array}\right]
$$

and the initial condition is $x^{\mathrm{T}}(0)=[0.5,-0.5,1]$. For comparison, the system is simulated with two different control schemes. In the first simulation, the passivity control in Reference [6] is applied to the system, and the 2-norm of the system state versus time is shown by the dash line in Figure 1. In the second simulation, control (27) in this paper is applied to the system with design parameters $P=I, \rho=1$ and $\varepsilon=0.1$, and the result shown by the solid line in Figure 1 . The figure clearly indicates that the decay rate from the new control (27) is exponential, and is much faster than the dash line resulting from the passivity control [6]. The system state under the new control virtually reaches the origin at around $12 \mathrm{~s}$.

\section{CONCLUSIONS}

Conventional controls for discrete-time homogeneous bilinear systems with (neutrally) stable dynamics can stabilize the system asymptotically, but not exponentially. In this paper, a new nonlinear control is constructed to stabilize the bilinear system exponentially. Achieving exponential stability has two important implications: (1) the system decay rate is much better improved than the previous non-exponential decay rate; (2) the controlled system becomes robust with respect to small parametric perturbations, as is shown in Reference [10].

However, the achieved exponential decay rate still has a limited exponent no matter how the control design parameters are chosen. A more challenging problem then is to construct a control that can produce any specified exponential decay rate, and this will be a subject to be pursued in the future.

\section{ACKNOWLEDGEMENTS}

The work in this paper is supported in part by the National Science Council of the Republic of China under the grant number NSC89-2213-E-002-087.

\section{REFERENCES}

1. Bruni C, Di Pillo G, Koch G. Bilinear systems: an appealing class of nearly linear systems in theory and applications. IEEE Transactions on Automatic Control, 1974; AC-19, 334-348.

2. Mohler RR. Nonlinear systems, Application to Bilinear Control, vol. 2, Prentice-Hall: Englewood Cliffs; NJ, 1990.

3. Ionescu T, Monopoli RV. On the stabilization of bilinear systems via hyperstability. IEEE Transactions Automatic Control, 1975; AC-20: 280-284.

4. Yang X, Chen LK. Stability of discrete bilinear systems with time-delayed feedback function. IEEE Transactions on Automatic Control, 1993; AC-38: 158-163.

5. Stepanenko Y, Yang X. Stabilizing controllers for discrete bilinear systems. International Journal of Robust and Nonlinear Control, 1996; 6: 855-867. 
6. Lin W, Byrnes CI. KYP lemma, state feedback and dynamic output feedback in discrete-time bilinear systems. System and Control Letters 1994; 23: 127-136.

7. Tarn TJ, Elliott DL, Goka T. Controllability of discrete bilinear systems with bounded control. IEEE Transactions on Automatic Control, 1973; AC-18: 298-301.

8. Vidyasagar M. Nonlinear System Analysis, Prentice-Hall: Englewood Cliffs, NJ, 1993.

9. Marsden JE, Hoffman MJ. Elementary Classical Analysis, W.H. Freeman and Company: New York, 1993.

10. Callier F, Desoer CA. Linear System Theory, Springer: New York, 1991. 